\title{
Stabilization of periodic orbits in a wedge billiard
}

\author{
Rodolphe Sepulchre* Manuel Gerard** \\ Department of Electrical Engineering and Computer Science \\ Université de Liège, Bât. B28 \\ B-4000 Liège, Belgium \\ r.sepulchre@ulg.ac.be, manuel.gerard@ulg.ac.be
}

\begin{abstract}
This paper introduces a stabilization problem for an elementary impact control system in the plane. The rich dynamical properties of the wedge billiard, combined to the relevance of the associated stabilization problem for feedback control issues in legged robotics make it a valuable benchmark for energy-based stabilization of impact control systems.
\end{abstract}

Keywords: periodic orbits; impact control; billiards; juggling.

\section{INTRODUCTION}

This paper is concerned with the stabilization of periodic orbits in the "wedge billiard" (or "planar juggler") illustrated in Figure 1.

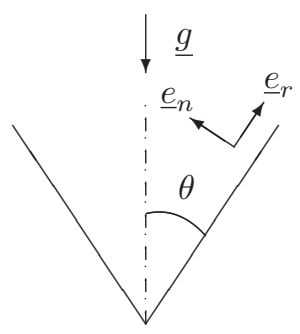

Fig. 1. The wedge billiard

A point mass (ball) moves in the plane under the action of a constant gravitational field. The ball undergoes elastic collisions with two intersecting edges, an idealization of the juggler's two arms. In the absence of control, the two edges form a fixed angle $\theta$ with the direction of gravity. Depending on the angle $\theta$, this conservative system exhibits a variety of dynamical phenomena, including an abundance of unstable periodic orbits. Rotational actuation of the edges around their fixed intersection point is used to stabilize one particular orbit of the uncontrolled system.

We view the wedge billiard stabilization as an interesting example for theoretical investigations of impact control problems encountered in legged robotics. The dynamics and control study of such mechanisms is rendered difficult by the inherently underactuated and intermittent nature of their

*This paper presents research partially supported by the Belgian Programme on Inter-university Poles of Attraction, initiated by the Belgian State, Prime Minister's Office for Science, Technology and Culture. The paper was completed while the first author was on sabbatical leave at Princeton University, Department of Mechanical and Aerospace Engineering. Discussions about the material of this paper with members of the department, especially P. Holmes, are gratefully acknowledged, as well as partial financial support from N. Leonard under US Air Force Grant F4962001-1-0382 and from E. Sontag under US Air Force Grant F49620-01-1-0063.

** Research Fellow of the Belgian National Fund for Scientific Research control. Starting with the pioneering work of Raibert and coworkers [12], the robotics community has nevertheless made dramatic advances over the last 15 years in building legged robots. Much of this research has focused on mechanisms that can sustain stable locomotion in the absence of actuation. Less research has been devoted to the "active control" of these mechanisms, that is the design of control laws that stabilize an otherwise unstable motion, with the notable exception of the work of Buehler, Koditschek and coworkers who pioneered the active stabilization of juggling machines [4, 13, 14, 5] . Buehler's planar juggler model is in fact the wedge billiard studied in the present paper for the particular angle $\theta=90 \mathrm{deg}$. The mirror law algorithm proposed in [4] has been tested experimentally with much success. In spite of its simplicity, a rigorous theoretical analysis of its stabilizing properties has apparently been elusive and originally motivated the present work. The design of stabilizing feedback laws for Buehler planar juggler has also been considered by Lynch [9] and by Brogliato and coworkers [19].

The stabilization problem considered in this paper is the simplest impact control problem beyond its one-dimensional version: the celebrated bouncing ball or line-juggler model, extensively studied in the literature (see for instance [7, $19,3,2])$. Considering the two-dimensional wedge billiard introduces few additional complexity in the model but raises stabilization questions that are more directly connected to the theoretical issues encountered in legged robotics and rhythmic tasks control. Most notably, the presence of a variety of unstable periodic orbits in the uncontrolled model and the fact that the stabilization of one particular periodic orbit can be viewed as a preliminary step towards the stabilization of a juggling "pattern", i.e. several balls stabilized on the same periodic orbit with a certain phase shift between them.

The stabilization of periodic orbits through impact control is naturally rephrased as the fixed point discrete-time stabilization of the corresponding Poincaré map. Stabilization of the planar juggler therefore leads to the stabilization of a three-dimensional discrete-time nonlinear system. The resulting model is nevertheless nonlinear and non-affine in the control, making it desirable (if not mandatory) to exploit the underlying conservative mechanical structure of the system in the design of stabilizing laws that have large basins of attraction and are robust to model uncertainties. Energybased or dissipativity-based methods for the stabilization of mechanical systems has been a very active research area over the last decade $[18,16,1,11]$. It is of interest to extend the applicability of these designs in the present context. 
From an implementation viewpoint, the stabilization of the discrete-time Poincaré map is only a preliminary step in the impact control stabilization problem. It indeed provides a discrete-time feedback law that prescribes the values of the control variables (edge angular position and velocity) at impact times based on the state of the ball at impact times. But the discrete control law must eventually be converted into a continuous-time control law for the actuated edges such as to make them produce the right discrete control values at impact times. Likewise, various continuous-time measurements can be integrated to produce a faithful reconstruction of the discrete states. These implementation issues are of importance for the robustness of the control law. They also raise important open questions such as the minimal feedback information required to stabilize a given periodic orbit. Of particular relevance for legged locomotion is the possibility to achieve stabilization in the absence of any feedback measurement (open-loop control) or by using the impact times as sole feedback information (rhythmic feedback).

Billiards have always been important objects in the study of dynamical systems. Beyond its relevance for robotic applications, the (uncontrolled) wedge billiard is a rich dynamical model leading to stabilization problems of various complexity. The wedge billiard was first studied in [8] for its relationship to self-gravitating systems in one dimension (the motion of $\mathrm{N}$ parallel sheets with uniform mass density). It was realized in this work and subsequent studies $[17,10]$ that the wedge billiard displays a variety of dynamical phenomena as a function of the angle $\theta$. For $\theta<45^{\circ}$, the phase space exhibits stable and chaotic behavior associated with periodic orbits of any period. For $\theta>45^{\circ}$, the motion appears completely chaotic. The value $\theta=45^{\circ}$ is very special and leads to a completely integrable system with a two-parameter family of unstable periodic orbits. The stabilization results of the present paper focus on this particular case which is simpler than the general case but illustrates most of the relevant issues of the problem.

The paper is organized as follows. In Section 2, we derive a dynamical model of the controlled wedge billiard. In Section 3 , we study the periodic orbits of the uncontrolled square wedge billiard, that is the particular case $\theta=45^{\circ}$. Stabilization control laws for this square billiard are then proposed in Section 4, using momentum control only, and in Section 5, using position control only. The mirror-law implementation of the proposed control laws is briefly discussed in Section 6.

\section{THE CONTROLLED WEDGE BILLIARD MODEL}

Periodic orbits of the four-dimensional wedge billiard dynamics will be studied via the three-dimensional discrete (Poincaré) map relating the state from one impact to the next one. The discrete-state vector, noted $x[k]$, will consist of continuous-time variables $x(t)$ evaluated at impact time $t[k]$. Because the continuous-time variables can be discontinuous at impact times, we use the notation $x^{-}(t[k])$ for pre-impact values and $x^{+}(t[k])$ for post-impact values. As a convention, the discrete-time state will denote post-impact values, that is $x[k]=x^{+}(t[k])$.

Let $\left(\underline{e}_{r}, \underline{e}_{n}\right)$ an orthonormal frame attached to the fixed point $O$ with $\underline{e}_{r}$ aligned with the impacted edge. Let $\underline{r}$ denote the position of the ball (unit mass point) and $\underline{v}=v_{r} \underline{e}_{r}+v_{n} \underline{e}_{n}$ its velocity. The total energy of the ball is

$$
E=\frac{1}{2}\left(v_{r}^{2}+v_{n}^{2}\right)-<\underline{r}, \underline{g}>
$$

Following [8], we use the state variables $V_{r}=\frac{v_{r}}{\cos \theta}, V_{n}=$ $\frac{v_{n}}{\sin \theta}$ and $E$, the discrete state vector being

$$
x[k]=\left(\begin{array}{c}
V_{r}^{+}(t[k]) \\
V_{n}^{+}(t[k]) \\
E^{+}(t[k])
\end{array}\right)
$$

In the absence of control, each edge forms an angle $\theta$ with the vertical, i.e. the direction of the constant gravitational field $g$. The discrete control vector $u[k]$ consists of the angular deviation $\mu(t[k])$ of the impacted edge at impact time $t[k]$ and its angular velocity $\dot{\mu}(t[k])$. It is assumed that the edge is not affected by the impacts, i.e. $\dot{\mu}^{-}(t[k])=\dot{\mu}^{+}(t[k])$.

The discrete wedge-billiard map is the composition of a (parabolic) flight map and an impact rule.

The flight map integrates the continuous-time equation of motion between two successive impact times, yielding

$$
x^{-}(t[k])=F_{1}(x[k-1], \mu[k-1], \mu[k])
$$

The impact map expresses post-impact variables as a (static) map of pre-impact variables and control in the form

$$
x[k]=F_{2}\left(x^{-}(t[k]), u[k]\right)
$$

We first review the derivation of the uncontrolled billiard map ([8]). The flight map is then entirely determined by the wedge geometry, that is by the parameter $\alpha=\tan \theta$. As shown in appendix, the flight map takes the analytical form $\mathcal{F}_{1}$

$$
\begin{aligned}
V_{n}^{-}(t[k+1]) & =-V_{n}[k] \\
V_{r}^{-}(t[k+1]) & =V_{r}[k]-2\left|V_{n}[k]\right| \\
\left(E^{-}(t[k+1])\right. & =E[k])
\end{aligned}
$$

when the impacts $k$ and $k+1$ occur on the same edge, and the analytical form $\mathcal{F}_{2}$

$$
\begin{aligned}
V_{n}^{-}(t[k+1])^{2} & =4 E[k]+\frac{2\left(1-\alpha^{2}\right)}{\left(1+\alpha^{2}\right)^{2}}\left(\left|V_{n}[k]\right|-V_{r}[k]\right)^{2}-V_{n}^{2}[k] \\
V_{r}^{-}(t[k+1]) & =\left|V_{n}[k]\right|-V_{r}[k]-\left|V_{n}^{-}(t[k+1])\right| \\
\left(E^{-}(t[k+1])\right. & =E[k])
\end{aligned}
$$

when the impacts $k$ and $k+1$ occur on two different edges. The map $\mathcal{F}_{1}$ is applied as long as the condition

$$
2 g y[k+1]=2 E[k]-V_{n}^{2}[k] \sin ^{2} \theta-\left(V_{r}[k]-2\left|V_{n}[k]\right|\right)^{2} \cos ^{2} \theta \geq 0
$$

is fulfilled. Otherwise, the map $\mathcal{F}_{2}$ is applied. This condition restricts the ball to impact above the intersection of the edges. The impact rule $\mathcal{I}$ adopted in this paper simply assumes 
that the tangential velocity is conserved and that the normal velocity is reversed :

$$
\begin{aligned}
& V_{r}^{+}(t[k])=V_{r}^{-}(t[k]) \\
& V_{n}^{+}(t[k])=-V_{n}^{-}(t[k])
\end{aligned}
$$

Collisions are thus perfectly elastic (leaving the energy conserved in the absence of control). The uncontrolled wedge billiard map is the composition of the flight maps $\mathcal{F}_{1}, \mathcal{F}_{2}$ and of the impact rule $\mathcal{I}$.

We now examine how control of the edges modifies the flight map and the impact rule. The angular momentum control $\dot{\mu}[k]$ has no effect on the wedge geometry. As a consequence, it leaves the flight map unchanged and only modifies the impact rule as

$$
V_{n}^{+}(t[k])=-V_{n}^{-}(t[k])+\frac{2}{\alpha} R(t[k]) \dot{\mu}(t[k])
$$

with $R(t[k])=\frac{r(t[k])}{\cos \theta}$ obtained from the energy equation (1).

In contrast, the angular position control $\mu(t[k])$ does not affect the impact rule but modifies the flight map. To avoid the complication of computing a new flight map, we introduce a simplification that leaves the flight map unchanged and captures the effect of the angular position control in a modified impact map. This simplification rests on the small control assumption

$$
|\mu|<<\theta
$$

and neglects the displacement of the impact point due to the angular deviation $\mu[k]$. As illustrated on Figure 2, this simplification amounts to assume that the impacts still occur on the uncontrolled wedge but that the angular control $\mu[k]$ rotates the normal and tangential directions of the impacted edge by an angle $\mu[k]$.

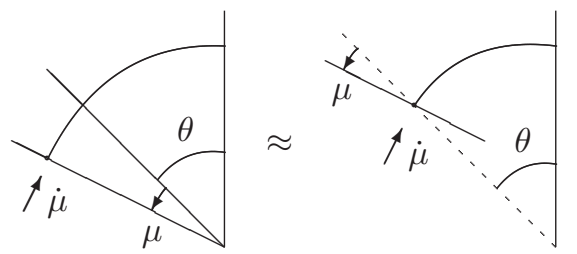

Fig. 2. The controlled wedge billiard (left) and a simplified model when $\mu$ is small (right)

With this simplification, the flight maps $\mathcal{F}_{1}, \mathcal{F}_{2}$ remain the flight maps of the uncontrolled billiard whereas the impact rule $\mathcal{I}$ becomes

$$
\begin{aligned}
M(\mu)\left(\begin{array}{c}
V_{r}^{+}(t[k]) \\
V_{n}^{+}(t[k])
\end{array}\right)= & \left(\begin{array}{cc}
1 & 0 \\
0 & -1
\end{array}\right) M(\mu)\left(\begin{array}{c}
V_{r}^{-}(t[k]) \\
V_{n}^{-}(t[k])
\end{array}\right)+ \\
& \left(\begin{array}{c}
0 \\
\frac{2}{\alpha} R(t[k])
\end{array}\right) \dot{\mu}(t[k])
\end{aligned}
$$

with $M(\mu)$ denoting the matrix

$$
M(\mu)=\left(\begin{array}{cc}
\cos \mu & \alpha \sin \mu \\
-\frac{\sin \mu}{\alpha} & \cos \mu
\end{array}\right)
$$

Note that (7) reduces to (6) when $\mu=0$.

Our simplified model neglects the displacement of the impact point due to the angular deviation $\mu$ but retains its "deflecting" effect on the velocity variables. Composing the flight maps $\mathcal{F}_{1}, \mathcal{F}_{2}$ and the impact rule (7), one obtains the discrete controlled billiard map

$$
\begin{gathered}
\left(\begin{array}{c}
V_{r}[k+1] \\
V_{n}[k+1]
\end{array}\right)=J(\mu)\left(\begin{array}{c}
V_{r}[k]-2\left|V_{n}[k]\right| \\
-V_{n}[k]
\end{array}\right) \\
+\frac{2}{\alpha}\left(\begin{array}{c}
-R[k+1] \alpha \sin \mu \\
R[k+1] \cos \mu
\end{array}\right) \dot{\mu}[k+1]
\end{gathered}
$$

for impacts $k$ and $k+1$ on the same edge, and the discrete controlled billiard map $\mathcal{B}$

$$
\begin{gathered}
\left(\begin{array}{c}
V_{r}[k+1] \\
V_{n}[k+1]
\end{array}\right)=J(\mu)\left(\begin{array}{c}
\left|V_{n}[k]\right|-V_{r}[k]-f_{1}[k] \\
f_{1}[k] \operatorname{sign}\left(V_{n}[k]\right)
\end{array}\right) \\
+\frac{2}{\alpha}\left(\begin{array}{c}
-R[k+1] \alpha \sin \mu \\
R[k+1] \cos \mu
\end{array}\right) \dot{\mu}[k+1]
\end{gathered}
$$

for impacts $k$ and $k+1$ on different edges, with

$$
\begin{aligned}
J(\mu) & =M(-\mu)\left(\begin{array}{cc}
1 & 0 \\
0 & -1
\end{array}\right) M(\mu) \\
& =\left(\begin{array}{cc}
\cos 2 \mu & \alpha \sin 2 \mu \\
\frac{\sin 2 \mu}{\alpha} & -\cos 2 \mu
\end{array}\right)
\end{aligned}
$$

and

$$
f_{1}[k]=\sqrt{4 E[k]+2 \frac{1-\alpha^{2}}{\left(1+\alpha^{2}\right)^{2}}\left(\left|V_{n}[k]\right|-V_{r}[k]\right)^{2}-V_{n}^{2}[k]}
$$

The energy update is

$$
\begin{aligned}
E[k+1]= & E[k]+\frac{1}{2} \frac{\alpha^{2}}{1+\alpha^{2}}\left(V_{n}[k+1]^{2}-V_{n}^{-}[k+1]^{2}\right)+ \\
& \frac{1}{2} \frac{1}{1+\alpha^{2}}\left(V_{r}[k+1]^{2}-V_{r}^{-}[k+1]^{2}\right)
\end{aligned}
$$

The analytical model (8)-(9) is exact when $\mu=0$ and is a good approximation of the controlled billiard under the small control assumption $|\mu|<<\theta$.

This simplified model is suitable for the analysis and design of stabilizing control laws of various periodic orbits of the uncontrolled billiard.

This will be illustrated in the next section for the special case $\alpha=1$ (or $\theta=45^{\circ}$ ).

\section{THE SQUARE WEDGE BILLIARD}

The square $(\alpha=1)$ elastic wedge billiard is a very special case of the general wedge billiard. Its analysis is of interest both because of its simplicity and because it possesses a twoparameter family of periodic orbits. As will be shown, all these orbits are open-loop unstable, making the stabilization non trivial even though stabilization will be achieved with arbitrarily small control.

The reason why the analysis of the square wedge billiard is considerably simplified is that in the absence of control, the 2 DOF motion decouples into two 1 DOF independent 
motions : in the (fixed) frame $\left(\underline{e}_{1}, \underline{e}_{2}\right)$ attached to the wedge, the dynamics of the mass point $\underline{x}=x_{1} \underline{e}_{1}+x_{2} \underline{e}_{2}$ satisfies

$$
\begin{aligned}
\ddot{x}_{i} & =-\frac{\sqrt{2}}{2} g \\
x_{i}(t) & =0 \Rightarrow x_{i}^{+}(t)=-x_{i}^{-}(t), i=1,2
\end{aligned}
$$

which directly yields the discrete map

$$
\begin{aligned}
V_{i}\left[k_{i}+1\right] & =V_{i}\left[k_{i}\right] \\
t\left[k_{i}+1\right] & =t\left[k_{i}\right]+\frac{2}{g} V_{i}\left[k_{i}\right]
\end{aligned}
$$

of an elastic bouncing ball or impact oscillator. Each solution of the impact oscillator is periodic of period $T_{i}=\frac{2 \sqrt{2} v_{n}^{i}}{g}$ where $v_{n}^{i}$ denotes the (constant) impact velocity $\left|\dot{X}_{i}\right|$. The periodic orbits of the square wedge billiard satisfy

$T_{1}=q T_{2}, \quad q \in \mathbb{N}$. In the rest of the paper, we only consider the case $q=1$. Such periodic orbits correspond to alternating impacts on the two billiard edges. They are therefore fixed points of the map $\mathcal{B}^{l}, \quad l \geq 1$, where $l$ is the total number of impacts during one period.

The simplification of the map $\mathcal{B}$ when $\alpha=1$ comes from the property

$\left|V_{n}[k+2]\right|=f_{1}[k+1]=\sqrt{4 E[k+1]-V_{n}[k+1]^{2}}=\left|V_{n}[k]\right|$

which renders the map $\mathcal{B}$ linear in the coordinates $Z[k]=$ $\left(V_{r}[k],\left|V_{n}[k]\right|,\left|V_{n}[k-1]\right|\right)$

$$
Z[k+1]=\tilde{B} Z[k]
$$

with

$$
\tilde{B}=\left(\begin{array}{ccc}
-1 & 1 & -1 \\
0 & 0 & 1 \\
0 & 1 & 0
\end{array}\right)
$$

Fixed points of the map $\tilde{B}$ are of the form $\bar{Z}=\left(0, \bar{V}_{n}, \bar{V}_{n}\right)$. They characterize a one-parameter family of periodic orbits, parametrized by their total energy $E=\frac{\bar{V}_{n}^{2}}{2}$.

All these period-one orbits are unstable because $\tilde{B}$ has an eigenvalue of (algebraic) multiplicity 2 on the unit circle.

Fixed points of the map $\tilde{B}^{2}$ are of the form $\bar{Z}=\left(\bar{V}_{r}, \bar{V}_{n}, \bar{V}_{n}\right)$. They characterize a two-parameter family of periodic orbits parametrized by their total energy $E=\frac{\bar{V}_{n}^{2}}{2}$ and the difference $\bar{V}_{n}-\bar{V}_{r}$. All these period-2 orbits are also unstable.

The factor $\frac{V_{n}-V_{r}}{g}$ has the convenient interpretation of a phase shift $\stackrel{g}{\phi}[k]=t[k]-t[k-1]$ between the two impact oscillators defining the billiard motion. This is a consequence of the formula

$$
V_{r}[k]-\left|V_{n}[k-1]\right|=-g(t[k]-t[k-1])=-g \phi[k]
$$

The phase variable $\phi[k]$ thus satisfies

$$
\phi[k+2]=\phi[k]+\frac{2}{g}\left(\left|V_{n}[k]\right|-\left|V_{n}[k-1]\right|\right)
$$

and can be substituted to the variable $V_{r}$ in the map $\tilde{B}^{2}$. An illustration of the period-two orbits of the square wedge is given on Figure 3 for a given total energy $E=\frac{\bar{V}_{n}^{2}}{2}$.

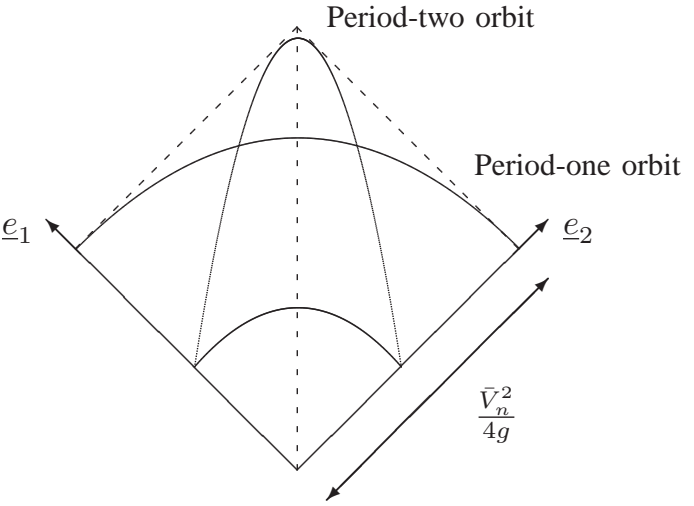

Fig. 3. An illustration of the period-one and period-two orbits of the square wedge billiard.

In the next two sections, it is implicitly assumed that impacts alternate on both billiard edges. This assumption is only made for simplicity and will be removed in the final version of the paper.

\section{ENERGY-BASED STABILIZATION OF THE SQUARE BILLIARD}

A very simple control strategy for the square billiard is to impose $\mu \equiv 0$ and to use angular momentum feedback control of each edge. Adding $\dot{\mu}$-control to the map $\tilde{B}^{2}$ and using the coordinates $\left(\phi[k], V_{n}[k], V_{n}[k-1]\right)$ yields the model

$$
\begin{aligned}
\phi[k+2] & =\phi[k]+\frac{2}{g}\left(\left|V_{n}[k]\right|-\left|V_{n}[k-1]\right|\right) \\
V_{n}[k+2] & =V_{n}[k]+2 R[k+2] \dot{\mu}[k+2] \\
V_{n}[k+1] & =V_{n}[k-1]+2 R[k+1] \dot{\mu}[k+1]
\end{aligned}
$$

The equilibrium characterized by $\left|V_{n}\right|=\bar{V}_{n}>0,0<\phi=$ $\bar{\phi}<\frac{2 \bar{V}_{n}}{g}$ is made asymptotically stable with the feedback control law

$$
\begin{aligned}
\dot{\mu}[k+2] & =-\frac{k_{P}}{\bar{R}}\left(V_{n}[k]-\bar{V}_{n}\right)-\frac{g k_{I}}{\bar{R}}(\phi[k]-\bar{\phi}) \\
\dot{\mu}[k+1] & =-\frac{k_{P}}{\bar{R}}\left(V_{n}[k-1]+\bar{V}_{n}\right)
\end{aligned}
$$

Exponential stability of the Jacobian linearization is ensured with mild conditions $0<k_{P}<1$ and $0<k_{I}<\frac{1-k_{p}}{2}$ on the (adimensional) design parameters $k_{P}$ and $k_{I}$.

The feedback control (13) has the standard structure of a proportional-integral control. With the interpretation of the wedge billiard as two coupled impact oscillators, the proportional feedback assigns the energy of each oscillator to a common energy level $\bar{V}_{n}^{2}$ whereas the integral term regulates the phase difference between the two oscillators.

The simple structure of the control law (13) leads itself to many variants that will be further analyzed in the final version of the paper. Of particular interest are its low-gain property and its rhythmic nature.

The low-gain property of the control law is that the size of the basin of attraction and the gain margin of the controller are increased as the control parameters $k_{P}$ and $k_{I}$ are lowered. As a consequence, the basin of attraction of the desired equilibrium can be made large and an arbitrarily low bound can be imposed on the magnitude of the control $|\dot{\mu}|$. 
The rhythmic nature of the control law (13) is due to the time equation

$$
t[k+2]-t[k]=\frac{2}{g}\left|V_{n}[k]\right|
$$

showing that the control law (13) can be rewritten as a function that uses the sequence of impact times $t[k]$ as sole feedback information.

\section{ENERGY-PRESERVING STABILIZATION OF THE SQUARE BILLIARD}

In the absence of angular momentum control $(\dot{\mu}=0)$, the controlled elastic wedge is conservative, the energy being conserved both during flight and through impacts. At fixed energy $E$, the square billiard has a one-parameter family of period-two orbits that are the fixed points of the map $\tilde{B}^{2}$.

We consider the problem of stabilizing one of these orbits using angular control actuation of one edge only. The controlled Poincaré map on the actuated edge then writes as :

$$
\left(\begin{array}{c}
V_{n}[k+2] \\
V_{r}[k+2]
\end{array}\right)=M(2 \mu)\left(\begin{array}{c}
V_{n}[k] \\
V_{r}[k]-2\left|V_{n}[k]\right|+2 \sqrt{4 E-V_{n}^{2}[k]}
\end{array}\right)
$$

Except for the value $\bar{V}_{r}=0$ at which the linearized system is not stabilizable, local asymptotic stability of the equilibrium characterized by $\bar{V}_{n}=\sqrt{2 E},-\sqrt{2 E} \leq V_{r}=\bar{V}_{r} \leq \sqrt{2 E}$ is achieved with the linear low-gain feedback

$\mu[k+2]=-\epsilon \operatorname{sign}\left(\bar{V}_{r}\right)\left(\left(V_{n}[k]-\bar{V}_{n}\right)-\epsilon \operatorname{sign}\left(\bar{V}_{n}\right)\left(V_{r}[k]-\bar{V}_{r}\right)\right), \epsilon>0$

where $\epsilon>0$ is a small control parameter. Lowering the gain $\epsilon>0$ results in a larger basin of attraction. Furthermore, the control law (15) can be saturated at an arbitrarily small constant magnitude $\bar{\mu}>0$ to validate the small angle assumption of the controlled model.

The square billiard model stabilized with angular control only is an elementary example of a conservative mechanical system which exhibits an asymptotically stable steady motion even though it lacks dissipation. Such mechanical systems are typically associated with nonholonomic constraints. Authors have previously observed that this situation also arises in piecewise holonomic systems, that is, mechanical systems that are smooth and holonomic except at discrete instants of impacts $[15,6]$. As noted in these references, such systems can be thought of as nonholonomically constrained in their overall motion in the sense that their configuration space is greater than the instantaneous dimension of their velocity space.

\section{MiRRor LAW IMPLEMENTATION}

To be implemented in a mechanical setup, the discrete-time control laws designed for the discrete-time impact model must be converted into continuous-time reference trajectories for the actuated edges.

Assuming a real-time measurement of the ball position, the mirror-law strategy proposed by Buehler and Koditschek [4] is a clever way to do so and is easily adapted to the present framework: after impact $n$ has occurred at time $t[n]$, the impacted edge is given the reference trajectory

$$
\mu(t)=F_{\dot{\mu}}(x[n]) \beta(t)+F_{\mu}(x[n]), t[n]<t \leq t[n+2]
$$

where $\beta(t)$ is the angular deviation of the ball at time $t$ with respect to the equilibrium edge angle and $x[n]$ denotes the state of the discrete-time impact system at impact $n$. By definition, the impact will occur when $\mu(t)=\beta(t)$, producing the discrete-time control law

$$
\mu[n+2]=\frac{F_{\mu}(x[n])}{1-F_{\dot{\mu}}(x[n])}
$$

and

$$
\dot{\mu}[n+2]=F_{\dot{\mu}}(x[n]) \dot{\beta}^{-}(t[n+2])
$$

The mirror-law continuous-time implementation thus approximates a discrete-time nominal design $\mu[n+2]=F_{\mu}(x[n])$ and $\dot{\mu}[n+2]=F_{\dot{\mu}}(x[n])$. The limited amplitude of $F_{\dot{\mu}}$ and the gain margin of the (low-gain) nominal design make it robust to the proposed approximate implementation.

\section{SIMULATION RESULTS}

The control law of Section 5 is now briefly illustrated by a simulation result. We choose to stabilize the periodic orbit characterized by $\left|\bar{V}_{n}\right|=\sqrt{11} \mathrm{~m} / \mathrm{s}, \bar{V}_{r}=-1.7 \mathrm{~m} / \mathrm{s}$. The initial condition is chosen as $V_{r}[0]=-\left|V_{n}[0]\right|=-3.25 \mathrm{~m} / \mathrm{s}$, which roughly corresponds to an initial vertical drop of the ball. Figure 4 compares the time evolution of the discrete variables $V_{n}$ and $V_{r}$ under the action of the energy-preserving control (15). The control law is compared in two different models:

1) approximate discrete map: this is the discrete map (14) used for the derivation of the control laws.

2) exact discrete map: this is the "true" Poincare map of the controlled wedge billiard, without the small angle approximation.
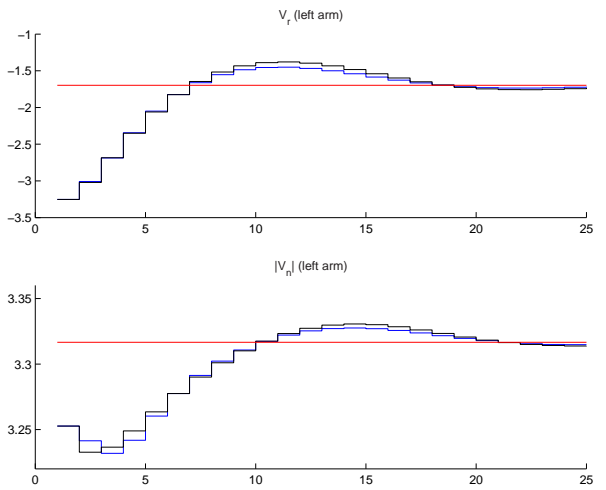

Fig. 4. Time evolution of the discrete variables $V_{r}$ and $V_{n}$

Figure 5 illustrates the trace of the trajectories.

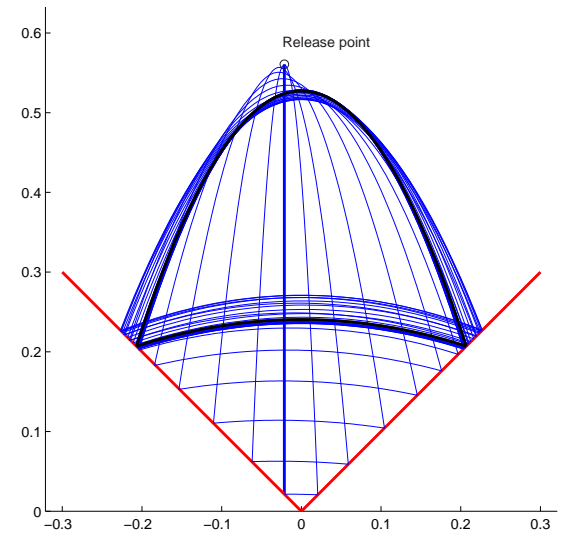

Fig. 5. Trace of the trajectories 


\section{CONCLUSION}

This paper has presented preliminary stabilization results for periodic orbits of the controlled wedge billiard, a model that generalizes the planar juggler model of Buehler and Koditschek and that we view as an interesting benchmark for impact control stabilization problems. The stabilization of periodic orbits of the square wedge billiard has been shown equivalent to the stabilization of two impact oscillators (or 1D bouncing balls) with a prescribed phase shift, leading to simple and robust feedback laws with large basins of attraction.

\section{REFERENCES}

[1] A.M. Bloch, N.E. Leonard, and J.E. Marsden, Controlled lagrangians and the stabilization of mechanical systems $i$ : The first matching theorem, IEEE Transactions on Automatic Control 45 (2000), no. 12, 22532270.

[2] M. Buehler and D.E. Koditschek, From stable to chaotic juggling : Theory, simulation, and experiments, IEEE International Conference on Robotics and Automation (Cincinnati, OH), 1990, pp. 1976-1981.

[3] M. Buehler, D.E. Koditschek, and P.J. Kindlmann, $A$ one degree of freedom juggler in a two degree of freedom environment, IEEE/RSJ Conf. Intelligent Systems and Robots (Tokyo, Japan), 1988, pp. pp. 91-97.

[4] , Planning and control of robotic juggling and catching tasks, International Journal of Robotics Research 13 (1994), no. 2, 101-118.

[5] R. R. Burridge, A. A. Rizzi, and D. E. Koditschek, Sequential composition of dynamically dexterous robot behaviors, International Journal of Robotics Research 18 (1999), no. 6, 534-555.

[6] M.J. Coleman and P. Holmes, Motions and stability of a piecewise holonomic system: the discrete chaplygin sleigh, Regular and Chaotic Dynamics 4 (1999), no. 2, $1-23$.

[7] P.J. Holmes, The dynamics of repeated impacts with a sinusodally vibrating table, Journal of Sound and Vibration 84 (1982), no. 2, 173-189.

[8] Lehtihet, H. E. Miller, and B. N., Numerical study of a billiard in a gravitational field, Physica 21D (1986), 93-104.

[9] K.M. Lynch and C.K. Black, Recurrence, controllability, and stabilization of juggling, IEEE Trans. on Robotics and Automation 17 (2001), no. 2, 113-124.

[10] V. Milner, J.L. Hanssen, W.C. Campbell, and M.G. Raizen, Optical billiards for atoms, Physical Review Letters 86 (2001), 1514-1517.

[11] R. Ortega, A. Loria, P.J. Nicklasson, and H. SiraRamirez, Passivity-based control of euler-lagrange systems, Spinger-Verlag, 1998.

[12] Marc H. Raibert, Legged robots that balance, Cambridge: MIT Press, 1986.

[13] A. A. Rizzi and D. E. Koditschek, Progress in spatial robot juggling, IEEE International Conference on Robotics and Automation (Nice, France), 1992, pp. 775-780.
[14] Further progress in robot juggling: The spatial two-juggle, IEEE International Conference on Robotics and Automation (Atlanta, GA), 1993, pp. 3:919-924.

[15] A. Ruina, Nonholonomic aspects of piecewise holonomic systems, reports on Matehmatical Physis 42 (1998), no. 1, 91-100.

[16] R. Sepulchre, M. Jankovic, and P. Kokotovic, Constructive nonlinear control, Spinger-Verlag, 1997.

[17] T. Szeredi and D.A. Goodings, Classical and quantum chaos of the wedge billiard. $i$. classical mechanics, Physical Review E 48 (1993), no. 5, 3518-3528.

[18] A.J. van der Schaft, L2-gain and passivity techniques in nonlinear control, Springer-Verlag, 2000.

[19] A. Zavala-Rio and B. Brogliato, On the control of a one degree-of-freedom juggling robot, Dynamics and Control 9 (1999), 67-90.

\section{APPENDIX}

Flight map $\mathcal{F}_{1}$

The flight time $t_{c}$ between two impacts on the same edge is the (positive) solution of the equation :

$$
\int_{0}^{t_{c}} V_{n}(\tau) d \tau=0
$$

which is found to be

$$
t_{c}=\frac{2}{g} V_{n}(0)
$$

Integrating once Newton's equations then yields

$$
\begin{aligned}
& V_{n}\left(t_{c}\right)=-V_{n}(0) \\
& V_{r}\left(t_{c}\right)=V_{r}(0)-2 V_{n}(0)
\end{aligned}
$$

which is the flight map $\mathcal{F}_{1}$.

\section{Flight map $\mathcal{F}_{2}$}

The flight time $t_{c}$ is the (positive) solution of the equation :

$$
\int_{0}^{t_{c}} V_{n}(\tau) d \tau+N(0)=0
$$

where $N(0)=\frac{n(0)}{\sin \theta}$ and $n($.$) is the component of the ball$ position relative to $\underline{e}_{n}$. The solution is :

$$
t_{c}=-\frac{V_{n}(0)}{g}+\frac{1}{g} \sqrt{V_{n}(0)^{2}-2 g N(0)}
$$

Integrating once Newton's equations gives at time $t_{c}$ :

$$
\begin{aligned}
V_{n}\left(t_{c}\right) & =V_{n}(0)+g t_{c} \\
& =\sqrt{V_{n}(0)^{2}-2 g N(0)} \\
V_{r}\left(t_{c}\right) & =V_{r}(0)-g t_{c}
\end{aligned}
$$

Equation (4) is obtained by summing (18) and (20) while Equation (4) is obtained from (19) and the energy relation:

$$
-2 g N(0)=4 E(0)-\frac{2}{1+\alpha^{2}}\left(V_{p}(0)^{2}+\alpha^{2} V_{q}(0)^{2}\right)
$$

where $V_{p}$ and $V_{q}$ are the tangential and normal (scaled) components of the velocity in the frame attached to the edge impacted at time 0 . 